\section{Blood brain barrier in a dish}

\section{By Lev Osherovich, Senior Writer}

Wisconsin researchers have used cultured human stem cells to engineer an artificial blood brain barrier. ${ }^{1}$ The technology could help companies identify drug candidates that readily enter the brain or that decrease blood brain barrier permeability.

The blood brain barrier (BBB) is a system of transporters and physical barriers in the endothelial cells of the brain vasculature that keeps most foreign molecules out of the brain. Breakdown of the BBB contributes to inflammatory and autoimmune diseases such as multiple sclerosis (MS) as well as certain forms of epilepsy.

Because the BBB arises from the complex interplay between endothelial cells, neurons and glia, it has proven difficult to recapitulate in vitro. Thus, testing for BBB-modulating drugs has been a slow, hit-or-miss process that requires testing in animals.

Previous in vitro systems for studying the BBB have suffered from numerous shortcomings, including poor scalability and inadequate separation of blood and brain compartments. Now, a team led by Eric Shusta, associate professor of chemical and
"When you take cells out of a mature brain and put them in cell culture out of their context, you lose a lot of the properties of the BBB. Instead, we're growing cells from the start to develop these properties."

-Eric Shusta,

University of Wisconsin-Madison
Indeed, a subset of cells grown in the endothelial cell-conditioned medium had higher levels of BBB-associated markers such as tight junction proteins and glucose transporters than cells grown in unconditioned medium.

Although some stem cells differentiated into endothelial cell precursors, other cells in the culture developed neuron-like characteristics and produced peptides including wingless-type MMTV integration site (WNT) family members that in turn promoted further development of the endothelial cells.

When purified and cultured on their own, the endothelial cell precursors from this mixed culture formed a layer of cells that resembled the endothelial layer of brain vascular tissue.

Next, Shusta's team set up an assay of BBB function in which the endothelial cells were cocultured with brain-derived astrocytes, with the two cell types separated by a porous filter. In this arrangement, the astrocytes provide soluble factors that direct the endothelial cells to form tightly associated layer with BBB-like properties.

Indeed, the artificial BBB made by Shusta's cells was markedly stronger than other in vitro BBB models. The cell layer had a high electrical resistance, indicating the tight junctions between the cells blocked the flow of ions and other soluble molecules from one side of the layer to the other.

"Our electrical resistance is substantially higher than any other systems, 50-100 times better than other human or animal models," said Shusta. "This is a measure of how tight the junctions are."

In an assay of small molecule transport, Shusta's artificial BBB excluded compounds known to be blocked from the brain and admitted molecules such as glucose that can readily cross the $\mathrm{BBB}$.

Shusta has filed to patent the technique. biological engineering at the University of Wisconsin-Madison, has overcome these problems by building an in vitro BBB from scratch using pluripotent stem cells as the starting point.

Shusta's team worked out a protocol to coax the stem cells into developing into an endothelial cell layer with BBB-like properties, including drug-pumping surface proteins and tight junctions between the cells (see Figure 1, "Building a better blood brain barrier").

"People have previously used primary cell culture, but this can't be scaled to the point of being useful for screening," said Shusta. "When you take cells out of a mature brain and put them in cell culture out of their context, you lose a lot of the properties of the BBB. Instead, we're growing cells from the start to develop these properties."

\section{Culture club}

Shusta's team set out to grow human pluripotent stem cells in an environment that mimics that of the developing brain. The researchers cultured human pluripotent stem cell lines in medium that had been exposed to cultured endothelial cells. As a result, the medium was laden with growth factors that encouraged endothelial cell development.
The patents are available for licensing from the Wisconsin Alumni Research Foundation, the technology transfer office of the University of Wisconsin.

\section{Barriers broken}

Shusta's technique opens the door to large-scale preclinical studies of drug penetration into the brain. Potential applications include screening for therapeutic candidates that readily cross the $\mathrm{BBB}$ or compounds that open the BBB to allow other therapeutic molecules to get across.

The in vitro system also could be used to study how the BBB opens up in neurological diseases such as MS, stroke and epilepsy. In these disorders, loss of $\mathrm{BBB}$ integrity is thought to contribute to brain damage, but the specific molecular players in pathological BBB opening have been hard to pin down.

Indeed, Shusta is now working on making brain endothelial cells from induced pluripotent stem cells from patients with MS and epilepsy.

According to Richard Daneman, research fellow in the Department of Anatomy at the University of California, San Francisco, prior in 


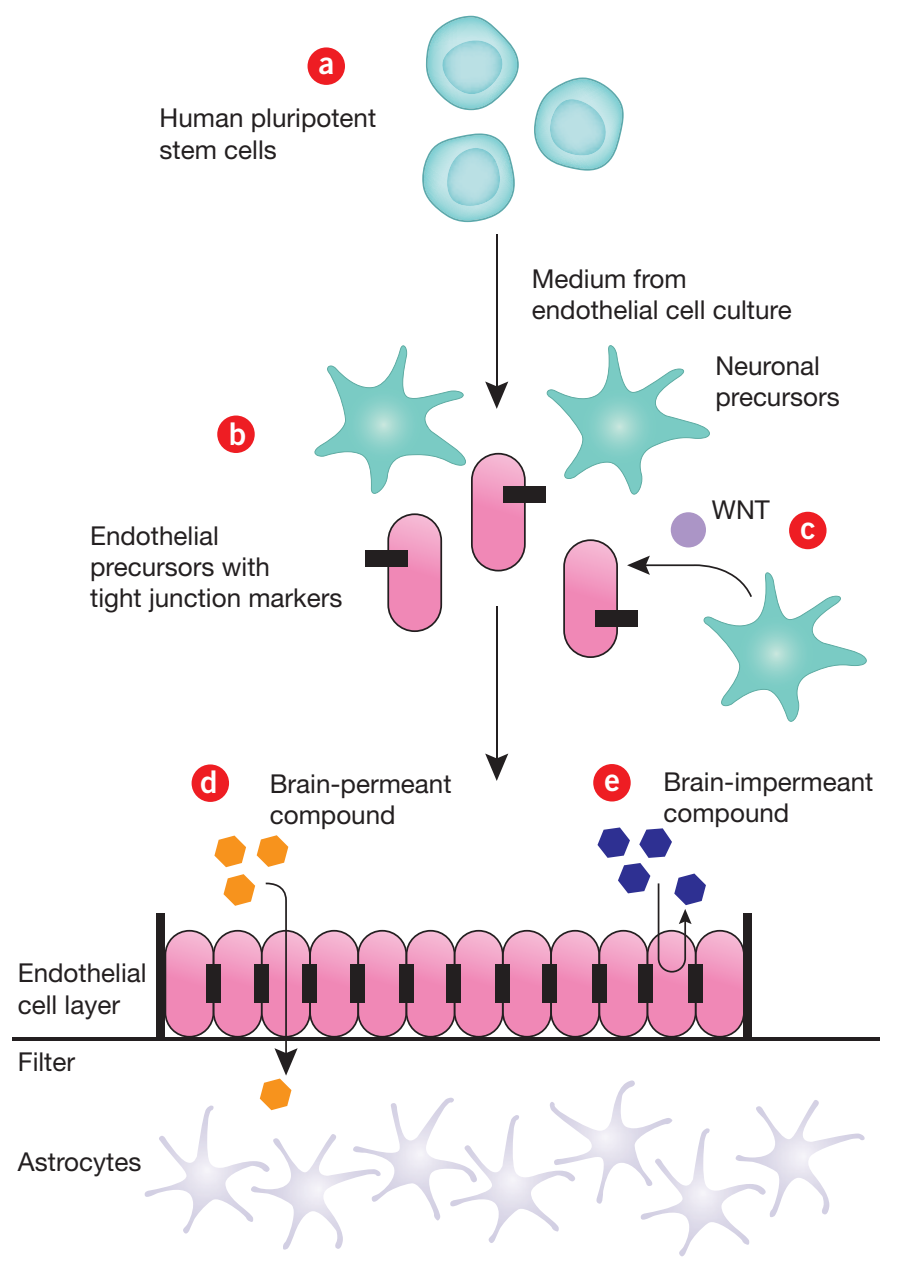

Figure 1. Building a better blood brain barrier. Lippmann et al. have modeled the blood brain barrier (BBB) in vitro using human pluripotent stem cells.

The technique involves treating undifferentiated human pluripotent stem cells [a] with cell culture medium that has previously been used to grow endothelial cells. The stem cells differentiate into a mixed population of neuronal and endothelial cell precursors [b] that express tight junction markers. The neurons in the mixed culture secrete developmental factors including wingless-type MMTV integration site (WNT) family members that guide the differentiation of endothelial cells $[\mathbf{c}]$. When cultured with rat astrocytes in an in vitro BBB assay, the resulting endothelial cells form tight junctions and show BBB activity, including the transport of brain-permeant compounds [d] and the exclusion of brain-impermeant compounds [e].

vitro $\mathrm{BBB}$ models required tradeoffs between physiological similarity to the human BBB, scalability and potency. Shusta's method scores high marks for all three, he said.

"The stem cell model here has several aspects that are better than all other models," said Daneman. "First, it's human—if you're trying to study drug delivery in humans, you want to use human tissue."

He noted that the method is also highly scalable and yields far more cells than was previously achieved with primary cell culture systems.
Daneman thinks the high potency of Shusta's cells arises from putting the stem cell precursors in an environment that resembles the embryonic brain.

In embryos, "these cells go through a lot of developmental stages to become brain endothelial cells," and Shusta's culture conditions mimic that environment, said Daneman.

\section{Smaller, better, faster}

Despite advantages in BBB potency and scalability, Shusta's stem cells may be harder to work with in an industrial setting than conventional BBB models that use transformed cells or primary cell culture because of the complexity of the culturing procedure, said Mohammad Kiani, professor and chair of mechanical engineering at Temple University.

Kiani also thinks the microfluidic platform for modeling the BBB and other functions of the microvasculature that he is developing will better replicate the role of blood flow at the BBB.

Kiani is collaborating with CFD Research Corp. (CFDRC) to develop a microfluidic platform for modeling the $\mathrm{BBB}$ and other functions of the microvasculature. Last month, CFDRC received a $\$ 1.3$ million grant from the NIH to further develop this technology, on which CFDRC holds patents.

Kiani said that growing endothelial and glial cells on separate parts of a microchip allows his team to study the effect of fluid flow on BBB functioning. He cited evidence that endothelial cells can regulate BBB activity in response to shear stress caused by blood flow.

Most in vitro BBB assays, including Shusta's, "are static systems where they look at cells or dyes going through a layer of cells. But that's not what happens in humans, where you have blood flow," said Kiani. "This makes a huge difference on the extravasation of particles and cells through the endothelium."

Thus, Kiani thinks in vitro systems that incorporate flow are "more physiological and in some cases fundamentally different than static conditions."

Shusta's assay of BBB activity involves traditional microtiter cell culture methods that do not easily scale up for high throughput screening. However, combining Shusta's cells with Kiani's microfluidic chambers could yield a more physiological and scalable model of the BBB.

Kiani, Shusta and Daneman agreed that testing the behavior of stem cell-derived endothelial cells in a microfluidic system could answer basic questions about BBB mechanisms.

"The idea behind flow is that sheer stress can regulate influx, but it's an open question as to whether flow opens or closes the BBB," said Shusta.

With microfluidic systems, "the amount of drug you need to screen is low and the total number of cells you might use goes down," said Shusta.

He cautioned that one concern about using microfluidic systems is that the small number of cells in such a chamber may show higher variability than larger cell cultures.

"You have to be careful about interpreting drug permeability data from a small number of cells because cells are not all uniform" in their BBB activity, Shusta noted.

Osherovich, L. SciBX 5(27); doi:10.1038/scibx.2012.694

Published online July 12, 2012 


\section{REFERENCES}

1. Lippmann, E.S. et al. Nat. Biotechnol.; published online June 24, 2012; doi:10.1038/nbt.2247

Contact: Eric V. Shusta, University of Wisconsin-Madison, Madison, Wis.

e-mail: shusta@engr.wisc.edu

Contact: Sean P. Palecek, same affiliation as above

e-mail: palecek@engr.wisc.edu
COMPANIES AND INSTITUTIONS MENTIONED

CFD Research Corp., Huntsville, Ala.

National Institutes of Health, Bethesda, Md.

Temple University, Philadelphia, Pa.

University of California, San Francisco, Calif.

University of Wisconsin-Madison, Madison, Wis.

Wisconsin Alumni Research Foundation,

Madison, Wis. 